\title{
Study on Inborn and Outborn Neonatal Admissions in Relation to Gestational Maturity in Neonatal Intensive Care Unit at a Tertiary Care University Hospital in Upper Egypt
}

\author{
Safwat M. Abdel-Aziz ${ }^{10}$ Enas A. Hamed ${ }^{2}{ }^{(1)}$ Amira M. Shalaby ${ }^{10}$ \\ ${ }^{1}$ Department of Paediatrics, Faculty of Medicine, Assiut University, \\ Assiut, Egypt \\ 2 Department of Medical Physiology, Faculty of Medicine, Assiut \\ Address for correspondence Enas Ahmad Hamed, MD, PhD, \\ Department of Medical Physiology, Faculty of Medicine, Assiut \\ University, Assiut 71516, Egypt (e-mail: eah3a2010@aun.edu.eg).
} University, Assiut, Egypt

J Child Sci 2021;11:e287-e295.

\begin{abstract}
Keywords

- admissions

- inborn

- neonatal intensive care unit

- newborns

- outborn

Neonatal morbidity and mortality rates indicate a country's socioeconomic status and the quality, and effectiveness of its health care system. This research aimed to identify the clinical pattern and causes of neonatal admission for inborn and outborn babies in a tertiary care university hospital and their outcomes. Over a year, this prospective hospital-based research was conducted in the neonatal intensive care unit (NICU) of Assiut Children's Hospital in Upper Egypt (January 1st to December $31^{\text {st }}, 2020$ ). Gender, birth weight, gestational age, postnatal age, delivery mode, delivery place, admission cause, hospital stay period, and neonatal outcomes were collected. A total of 1,638 newborns were admitted; 930 (56.8\%) were preterm and 708 (43.2\%) full-term. Inborn admissions were 1,056 (64.5\%) and outborn 582 (35.5\%). The majority of inborn admissions were preterm 726 (68.8\%), and outborn were full-term 378 (64.9\%). The commonest admission causes among inborn and outborn preterm infants were respiratory distress syndrome (84.3\%) and congenital intestinal obstruction (22.5\%), respectively, while multiple congenital anomalies were the commonest cause for admission among both inborn and outborn full-term babies. The mortality rate was 708 (43.2\%), higher among inborn (50\%) versus outborn (30.9\%). The leading cause of death was respiratory distress syndrome among premature inborn with case fatality rate of (56.9\%) and multiple congenital anomalies among premature outborn (60\%), as well as inborn (67.4\%), and outborn (42.6\%) full-term neonates. In conclusion, the neonatal mortality rate was high among studied cases. Morbidity and mortality of respiratory distress syndrome and congenital anomalies were alarmingly high. Therefore, all health care providers must devote a considerable effort to improve health care delivered to these neonates.
\end{abstract}

received

May 21, 2021

accepted after revision

August 30, 2021
DOI https://doi.org/

10.1055/s-0041-1736478.

ISSN 2474-5871.
(C) 2021. The Author(s).

This is an open access article published by Thieme under the terms of the Creative Commons Attribution License, permitting unrestricted use, distribution, and reproduction so long as the original work is properly cited. (https://creativecommons.org/licenses/by/4.0/)

Georg Thieme Verlag KG, Rüdigerstraße 14, 70469 Stuttgart, Germany 


\section{Introduction}

Since it is vulnerable to high mortality and morbidity, neonatal time (first 28 days of life) is important in life. ${ }^{1}$ Over the last few decades, advances in neonatal care greatly improved the newborns' survival rate, especially premature newborns. So, neonatal admissions and resulting neonatal diseases are rising. ${ }^{2}$ Neonatal intensive care unit (NICU) admissions place a significant financial and social burden on health care facilities and families, also, disease patterns differ from location to location and with time, even in the same place. ${ }^{3}$ Every year, approximately nine million children die in the perinatal and neonatal period across the world, with nearly all of the mortality occurring in developing countries (98\%). Neonatal deaths are responsible for 40 to $70 \%$ of all infant deaths. ${ }^{4}$ Evidence on neonatal mortality in developing countries is limited, and risks are poorly defined. The neonatal mortality rate (NMR) in Egypt in 2013 was 11.8 per 1,000 live births- approximately $54 \%$ of all mortality in children under the age of 5 years. ${ }^{5}$

Based on WHO statistics, there were 2.5 million infant deaths worldwide in the first month after birth; 7,000 neonates died per day, with about one-third dying in the first 24 hours of life and approximately $75 \%$ in the first 7 days after birth. Moreover, the rate of neonatal death decreased more slowly, compared with that of children aged 1 to 59 months. ${ }^{6}$

Worldwide, premature birth (27\%), infection (26\%), perinatal asphyxia (23\%), and congenital malformations (7\%) are the leading causes of neonatal deaths. ${ }^{7}$ Other authors reported low birth weight, prematurity, sepsis, low Apgar scores, respiratory distress syndrome (RDS), low socioeconomic status, delivery by cesarean section, and postnatal age on admission are risk factors linked with mortality in the neonatal period. ${ }^{8}$ Researchers reported that the primary reasons for $78 \%$ of all non-malformation-related mortality in preterm babies were RDS, severe perinatal asphyxia, and infections. Other reasons for newborn deaths were bronchopulmonary dysplasia (4\%), intraventricular hemorrhage (3\%), pneumothorax (2\%), and necrotizing enterocolitis $(2 \%) .{ }^{9}$ In developed countries, newborns mostly die due to unpreventable conditions, such as congenital malformations, while most neonatal deaths in developing countries are due to preventable reasons, such as birth asphyxia, infections, and prematurity. ${ }^{10}$ Evidence-based information on neonatal morbidities, mortalities, and interventions, particularly facility-based care are critical for implementing newborn preventive health strategies to decrease neonatal deaths.

As a result, this research aimed to evaluate the profile of inborn and outborn newborns admitted to a tertiary care referral and teaching hospital in Upper Egypt, as well as to evaluate their outcomes.

\section{Methods}

This hospital-based prospective observational study was performed over a period of 1 year from January 01, 2020 to December 31, 2020 in the NICU of Assiut University
Children's Hospital. This hospital provides health care services for people in the Assiut governorate and its surrounding communities in the urban and rural areas in Upper Egypt. It is equipped with all facilities toward being one of the main referral hospitals in Upper Egypt. The NICU provides services for approximately 145 newborns per month with a total of 50 incubators and has facilities dedicated for outborn and inborn care. It serves the obstetrics department of Women's Health Hospital which is one of the Assiut University Hospitals and their delivered newborns needing admission (inborn). Furthermore, it receives outpatients, who are referrals from primary health centers, or from a diversity of places (outborn). Although our hospital serves five governorates in Upper Egypt-each one including approximately 2 million people-there are no specific data for live births and population in the surrounding referral region, due to the poor registration system in such places,

\section{Study Population and Sampling Size}

All inborn and outborn newborns that were admitted to the NICU during the research period were included in this study.

\section{Data Collection Tools}

Detailed information for each of the neonatal admissions was taken including gender, delivery mode, delivery place, gestational age, birth weight, age at admission to NICU, length of hospital stay, maternal risk factors, causes for admission, reasons for neonatal mortality, and outcome of admitted cases. The hospital has a standard protocol for the diagnosis and management of neonatal conditions. The diagnosis was mostly clinically supported by laboratory investigations, radiological imaging, and echocardiography when needed.

In our hospital, almost all neonatal conditions have been protocolized for diagnosis and management of different neonatal diseases as neonatal sepsis, necrotizing enterocolitis, respiratory distress, neonatal seizures, and hyperbilirubinemia. In our neonatal unit, plain X-rays, transcranial ultrasonography (US), computed tomography (CT), and magnetic resonance imaging (MRI) scan, echocardiography, barium studies, and electroencephalography (EEG) can be done.

\section{Ethical Issues}

The research was approved by the Local Ethical Committee Institutional Review Board (IRB) of Assiut University Hospital, Assiut, Egypt (IRB: 17100776, December 29, 2019) that was according to the Declaration of Helsinki. Informed written consent was obtained from mothers or guaranteed of every participant before inclusion, after explaining the research aim to them at admission.

\section{Statistical Analysis}

Data were analyzed by Statistical Package for Social Sciences version 23 (IBM SPSS, IBM Corp., Armonk, New York, United States). Values were presented as mean \pm standard deviation of number (\%) as appropriate. During univariate analysis, the categorical values were tested with Pearson Chi-square and Fisher exact test as appropriate. $p$-Values less than 0.05 were considered statistically significant. 
Table 1 Demographic features of the studied neonates $(n=1,638)$

\begin{tabular}{|l|l|l|l|}
\hline \multicolumn{2}{|l|}{ Characteristics } & Frequency (\%) & Significance \\
\hline \multirow{2}{*}{$\begin{array}{l}\text { Gestational } \\
\text { maturity }\end{array}$} & Preterm & $930(56.8 \%)$ & $p<0.0001$ \\
\cline { 2 - 4 } & Full term & $708(43.2 \%)$ & \\
\hline \multirow{2}{*}{ Sex } & Males & $865(52.8 \%)$ & $p=0.023$ \\
\cline { 2 - 4 } & Females & $773(47.2 \%)$ & \\
\hline $\begin{array}{l}\text { Mode of } \\
\text { delivery }\end{array}$ & CS & $1,347(82.2 \%)$ & $p<0.0001$ \\
\cline { 2 - 4 } & Vaginal & $291(17.8 \%)$ & \\
\hline \multirow{2}{*}{$\begin{array}{l}\text { Place of } \\
\text { delivery }\end{array}$} & Inborn & $1,056(64.5 \%)$ & $p<0.0001$ \\
\cline { 2 - 4 } & Outborn & $582(35.5 \%)$ & \\
\hline \multirow{2}{*}{ Outcome } & Survival & $930(56.8 \%)$ & $p<0.0001$ \\
\cline { 2 - 4 } & Died & $708(43.2 \%)$ & \\
\hline
\end{tabular}

Note: Significance was made using Person Chi-square test.

\section{Results}

A total of 1,638 neonates were admitted during the research period. Admitted preterms were significantly higher than full-terms ( 56.8 vs. $43.2 \%, p<0.0001$ ); males were higher than females ( 52.8 vs. $47.2 \%, p=0.023$ ), those delivered by CS (cesarean section) were higher than spontaneous vaginal delivery ( 82.2 vs. $17.8 \%, p<0.0001$ ) and inborn were higher than outborn ( 64.5 vs. $35.5 \%, p<0.0001)$. The mortality rate among all admitted newborns was $43.2 \%$ (-Table 1).

- Table 2 shows the baseline characteristics of the studied preterm and full-term babies. Preterm babies represented 930 (56.8\%) of them (726, 78.1\% inborn, $204,21.9 \%$ outborn), male babies were 490 (52.7\%), and majority of cases were delivered by CS 762 (81.9\%). With regards to weight on admission and gestational age, $75.3 \%$ of cases were in the category of 1 to $1.5 \mathrm{~kg}$, and $74.9 \%$ in the category of 28 to 32 weeks. Regarding age at admission, majority of preterm cases were admitted in first day of life 740 (79.5\%). Based on duration of hospital stay, more than half of the cases, 511 (54.9\%) stayed for 7 to 15 days. According to maternal risk factors, preeclampsia was considered the most common risk factor among studied preterm cases. There were statistically significant differences between inborn and outborn preterm newborns in the mode of delivery, postnatal age at admission, duration of hospital stay, and maternal risk factors ( $p=0.001, p=0.001, p=0.001$ and $p=0.007$, respectively). Full-term babies were 708 (43.2\%), among which 330 (46.6\%) were inborn and 378 (53.4\%) were outborn, male babies were 375 (53\%), and majority of the cases were delivered by CS $(585,82.6 \%)$. With regard to the weight on admission; majority of cases 610 (86.2\%) were in the category of 2.5 to $<4 \mathrm{~kg}$. Considering the age at admission, about one-half of cases $(356,50.3 \%)$ were admitted within the first day of life. Based on the hospital stay duration, 408 (57.6\%) stayed for 7 to 15 days. According to maternal risk factors, premature rupture of membranes (PROM) was the most common risk factor among full-term cases. There were statistically significant differences between inborn and outborn full-term newborns in postnatal age on admission, duration of hospital stay, and maternal risk factors $(p=0.001, p=0.001$ and $p=0.001$, respectively).

Commonest causes of admission among preterm babies were RDS ( $n=643,69.1 \%$ ), followed by congenital intestinal obstructions (duodenal/ileal/colonic atresia, Hirschprung's disease, meconium ileus, meconium plugs syndrome, intussusceptions, and neonatal small left colon syndrome) $(n=58$, $6.2 \%)$, neonatal jaundice $(n=42,4.5 \%)$, sepsis $(n=38,4.1 \%)$, multiple congenital anomalies as chromosomal abnormalities, musculoskeletal deformities with congenital heart or renal defects $(n=36,3.9 \%)$, and transient tachypnea of newborn $(n=36,3.9 \%)$. The inborn were significantly higher than outborn preterm newborns in RDS, transient tachypnea of newborn, neural tube defect, perinatal asphyxia and hypoglycemia and infant of diabetic mother (IDM) $(p=0.001$ for all). Meanwhile the outborn were significantly higher than inborn preterm newborns in congenital intestinal obstruction, neonatal jaundice, sepsis, multiple congenital anomalies, pneumonia, and neonatal seizures $(p=0.001$ for all).

Among full-term babies, the commonest cause for hospital admission was multiple congenital anomalies $(n=180$, $25.4 \%$ ), followed by neonatal jaundice ( $n=108,15.3 \%)$, congenital intestinal obstruction $(n=108,15.3 \%)$, transient tachypnea of newborn $(n=66,9.3 \%)$, neural tube defects $(n=48,6.8 \%)$, and sepsis $(n=44,6.2 \%)$. The inborn were significantly higher than outborn full-term newborns in transient tachypnea of newborn, perinatal asphyxia and hypoglycemia and IDM, meconium aspiration syndrome ( $p=0.001$ for all). On the other hand, the outborn were significantly higher than inborn full-term newborns in congenital intestinal obstruction, neonatal jaundice, sepsis, neonatal seizures, and thrombocytopenia ( $p=0.001, p=0.001$, $p=0.006, p=0.001$, and $p=0.032$, respectively) ( - Table 3 ).

Number of deaths in preterm infants was 462 . The cause of deaths with a case fatality rate of all deaths were mostly RDS $78.4 \%$, followed by sepsis (5.0\%), multiple congenital anomalies $(4.8 \%)$, neural tube defects $(2.8 \%)$, congenital intestinal obstruction (2.4\%), pneumonia (2.2\%), perinatal asphyxia (1.7\%), transient tachypnea of newborn (1.3\%), and neonatal jaundice (1.1\%). The causes of deaths in inborn were significantly higher than outborn preterm newborns in RDS, neural tube defect, and transient tachypnea of newborn $(p=0.001$ for all). Whereas the causes of deaths in outborn were significantly higher than inborn preterm newborn in sepsis, multiple congenital anomalies, congenital intestinal obstruction, and pneumonia $(p=0.001, p=0.001, p=0.003$ and $p=0.001$, respectively). The number of deaths in full-term infants was 246 . The causes of deaths were mostly multiple congenital anomalies (39.8\%), followed by congenital intestinal obstruction (13.0\%), perinatal asphyxia (9.8\%), sepsis (8.5\%), meconium aspiration syndrome (7.7\%), neural tube defects (7.3\%), pneumonia (6.1\%), neonatal jaundice (4.1\%), hypoglycemia and IDM (1.2\%), transient tachypnea of newborn $(0.8 \%)$, neonatal seizures $(0.8 \%)$, and thrombocytopenia $(0.8 \%)$. The causes of deaths in inborn were significantly higher than outborn full-term newborns in multiple 


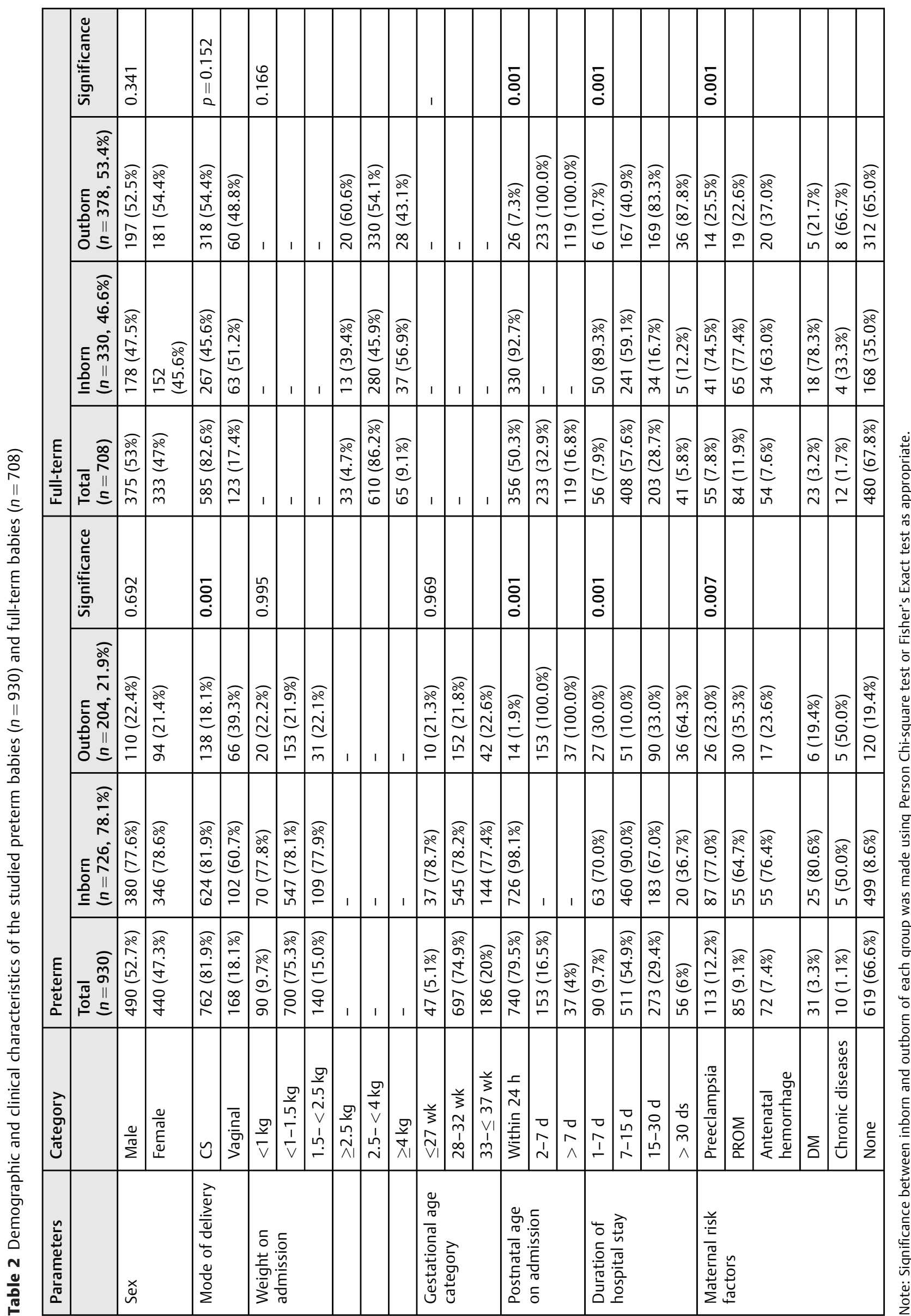




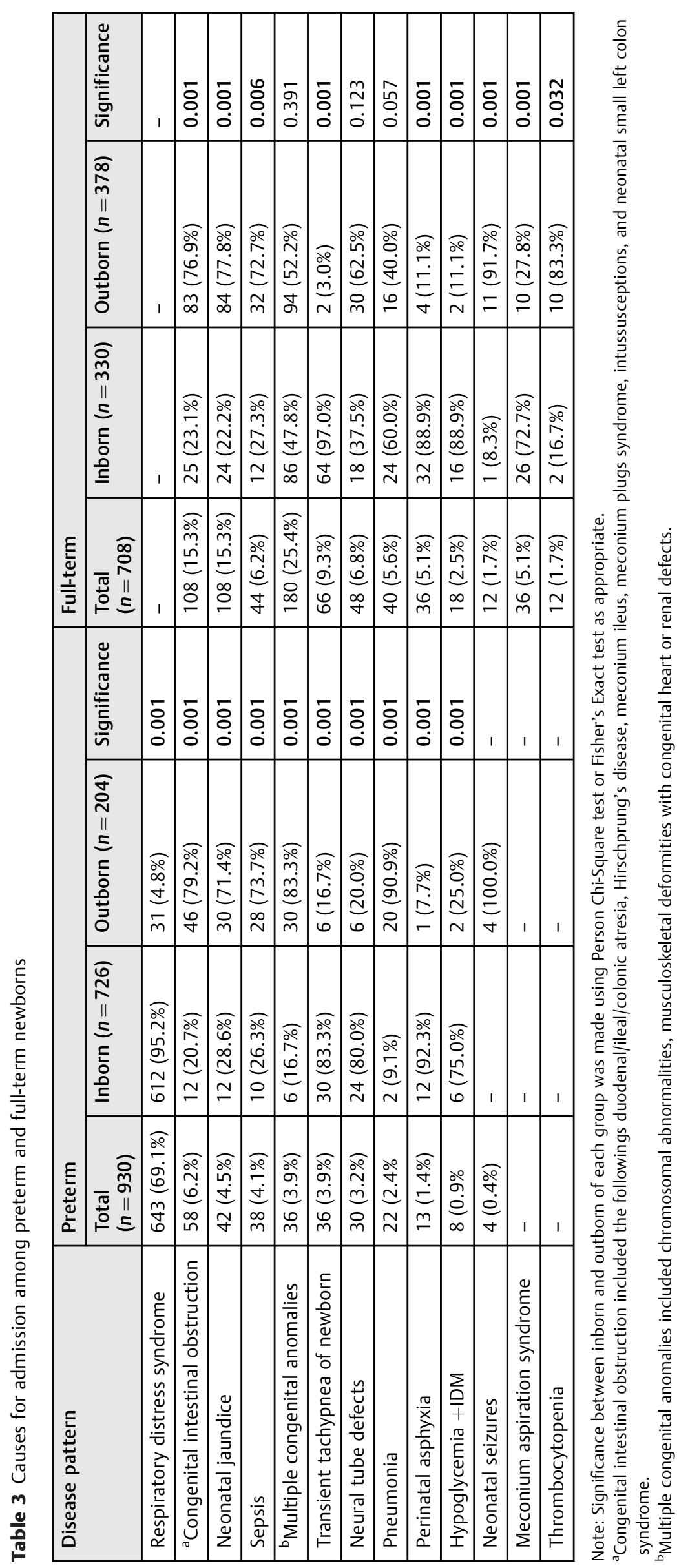


congenital anomalies, perinatal asphyxia, and meconium aspiration syndrome $(p=0.005, p=0.001$ and $p=0.044$, respectively). However, the causes of deaths in outborn were significantly higher than inborn full-term newborn in sepsis and neonatal jaundice ( $p=0.001$ for both) ( - Table 4 ).

- Table 5 clarifies the distribution of congenital anomalies among the studied neonates.

\section{Discussion}

Morbidity and mortality data obtained from regular registration are beneficial for both health care providers and researchers to propose interventions for prevention, treatment, and adjusting the quality of care. In the present study, preterm babies represented the majority of neonatal admissions, ( $56.8 \%$ of which $78.1 \%$ were inborn and $21.9 \%$ outborn) while full-term babies represented $43.2 \%$ (46.6\% inborn and $53.4 \%$ outborn). The majority of admitted neonates were inborn (64.5\%), with an inborn to outborn ratio of 1.8:1. In Egypt, at Zagazig University Children Hospital, Abd el aziz et $\mathrm{al}^{11}$ reported that preterm were higher than full-term neonates ( 55.9 vs. $44.1 \%$ ) and inborn were higher than outborn neonates ( 83.1 vs.16.9\%). A study done by Fahmy et al ${ }^{12}$ at Cairo University Hospital showed that majority of inborn admitted were premature (72.5\%) while majority of outborn admitted were full-term (67.7\%). On the contrary, at Addis Ababa, Ethiopia, Tekleab et $\mathrm{al}^{13}$ reported that $39.8 \%$ of neonatal admission were preterm while majority (60.2\%) were full-term.

Our results showed that, most cases delivered by CS (82.2\%), which matched with the Egypt demographic and health survey (EDHS) $2014,{ }^{14}$ that reported $87 \%$ of all live births were delivered in a hospital and slightly more than half of births (52.0\%) were delivered by CS. On the contrary, Bokade and Meshram ${ }^{15}$ and Verma et al ${ }^{16}$ showed different results as 31.9 and $30.4 \%$ were born by CS, respectively. This may be explained by increasing emergency CS in tertiary Gynecology/Obstetric hospitals that receive high-risk pregnant women.

In this study, males were more than females (52.8 vs. $47.2 \%$ ) which was similar to others. ${ }^{17,18}$ This could be a reflection of some cultural beliefs where the male baby is preferred to female and more likely to be paid attention when he was ill.

Our results showed that the majority of neonatal admissions were in the first day of life (79.5\% preterm, and $50.1 \%$ full-term). Similarly, Seboka et al $^{19}$ showed in their research that most newborns were admitted during first 24 hours following birth (76\%). Also, our results were consistent with others. ${ }^{20}$ As this is a transition from intrauterine to extrauterine life, majority of neonatal issues may arise within the first 24 hours after birth. ${ }^{20}$

The commonest cause for admission in this study among preterm babies was RDS (69.1\%), followed by congenital intestinal obstruction (6.2\%), neonatal jaundice (4.5\%), and sepsis (4.1\%). Furthermore, the commonest cause for hospital admission among full-term babies was multiple congenital anomalies (25.4\%), followed by neonatal jaundice (15.3\%),

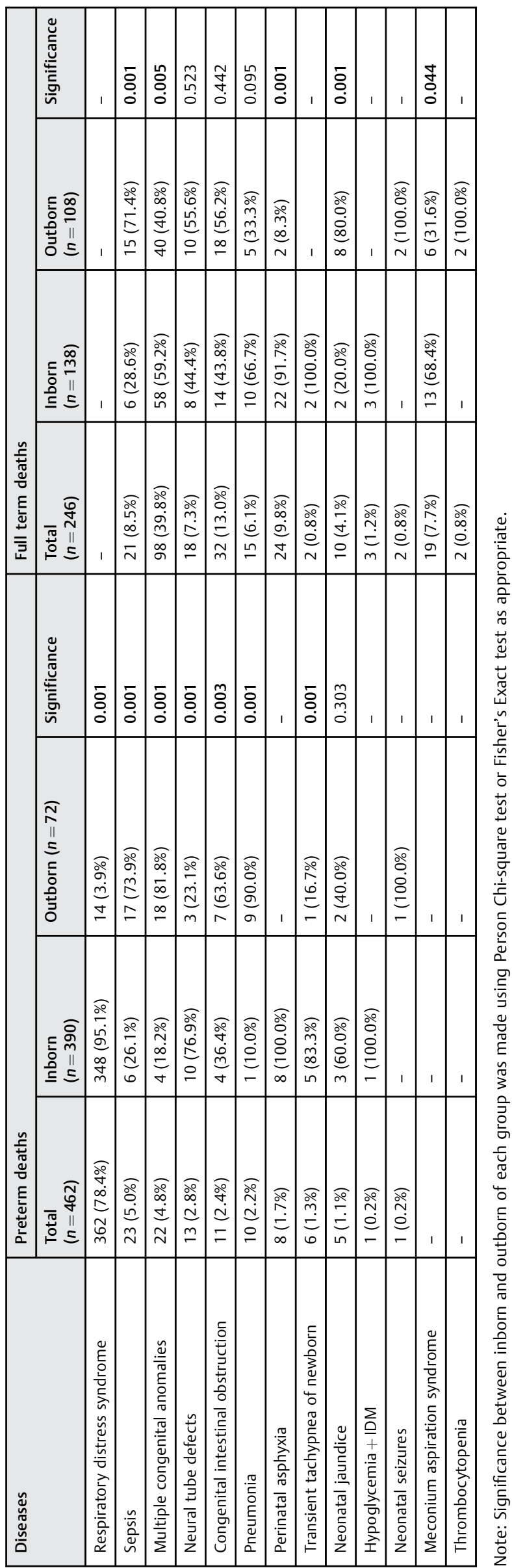


Table 5 Congenital anomalies distribution among the studied neonates

\begin{tabular}{|c|c|c|c|}
\hline \multicolumn{2}{|l|}{ Type of congenital anomaly } & $\begin{array}{l}\text { Preterm } \\
(n=930)\end{array}$ & $\begin{array}{l}\text { Full term } \\
(n=708)\end{array}$ \\
\hline \multicolumn{2}{|c|}{${ }^{\mathrm{a} C}$ Congenital intestinal obstructions } & $58(6.2 \%)$ & $108(15.3 \%)$ \\
\hline \multicolumn{2}{|c|}{ Neural tube defects ${ }^{\mathrm{b}}$} & $30(3.2 \%)$ & $48(6.8 \%)$ \\
\hline \multirow[t]{5}{*}{ Multiple congenital anomalies } & $\begin{array}{l}\text { Musculoskeletal plus } \\
\text { Heart defects }\end{array}$ & $12(1.3 \%)$ & $56(7.9 \%)$ \\
\hline & GIT anomalies plus abdominal defects & $11(1.2 \%)$ & $66(9.3 \%)$ \\
\hline & CNS anomalies plus skeletal defects plus facial anomalies & $8(0.9 \%)$ & $45(6.4 \%)$ \\
\hline & Genetic syndromes plus heart and urogenital defects & $3(0.3 \%)$ & $5(0.7 \%)$ \\
\hline & $\begin{array}{l}\text { Down syndrome } \\
\text { plus, heart defects }\end{array}$ & $2(0.2 \%)$ & $8(1.1 \%)$ \\
\hline Total & & $124(13.3 \%)$ & $336(47.5 \%)$ \\
\hline
\end{tabular}

Abbreviations: CNS, central nervous system; GIT, gastrointestinal tract.

${ }^{a}$ Congenital intestinal obstruction included the followings duodenal/ileal/colonic atresia, Hirschprung's disease, meconium ileus, meconium plugs syndrome, intussusceptions, and neonatal small left colon syndrome.

${ }^{\mathrm{b}}$ Neural tube defects include hydrocephalus, meningomyelocele, cerebral, and cerebellar anomalies.

congenital intestinal obstruction (15.3\%), transient tachypnea of newborn (9.3\%), neural tube defects (6.8\%), and sepsis (6.2\%). A similar study carried in the NICU at Zagazig University Children Hospital, Egypt, reported that the commonest diagnosis during NICU admission was RDS (21.1\%) followed by surgical causes (14.6\%), late onset sepsis (12.2\%), and congenital pneumonia (8.5\%). ${ }^{11} \mathrm{~A}$ recent study conducted in Qena (Upper Egypt) reported that the incidence of respiratory distress in neonates was $46.5 \%$. Mean gestational age of the newborns was $34.49 \pm 3.31$ weeks. Commonest causes of respiratory diseases were RDS (49.6\%) transient tachypnea of newborn (22.0\%), neonatal pneumonia (17.2\%) and meconium aspiration syndrome (6.2\%). ${ }^{21}$ Elizabeth and Oyetunde ${ }^{22}$ reported that congenital anomalies were the most frequent cause of morbidity among studied neonates (7.22\%)musculoskeletal defect (33.33\%) being the commonest congenital anomaly amongst them. Eze et $\mathrm{al}^{23}$ showed that the main neonatal morbidities were prematurity and its complications-neonatal jaundice, perinatal asphyxia, and neonatal sepsis. Prematurity complications and perinatal asphyxia were responsible for approximately one-third of NICU admissions each and were the most predominant causes of neonatal morbidity. A study done in Nigeria showed premature low birth weight (54.8\%), birth asphyxia (19.2\%), respiratory distress (6.7\%), sepsis (5.3\%), congenital malformations (1.2\%), and neonatal jaundice $(1.1 \%)$ as main reasons for admission. ${ }^{20}$ Research in Pakistan ${ }^{24}$ showed that preterm low birth weight newborns (24.6\%), meconium aspiration syndrome (15.2\%), birth asphyxia (17.0\%), sepsis (19.9\%), RDS (18.9\%), and neonatal jaundice (9.44\%) were the common causes for admission.

The overall neonatal mortality in this study was 708 (43.2\%). In preterm cases, 462 (49.7\%) died, with inborn admissions accounting for majority of deaths (53.7\%). On the other hand, $34.7 \%$ of admitted full-term cases died, inborn admissions accounting for deaths in majority (41.8\%). These results were higher than other studies conducted in Egypt which showed that the NMRs among cases admitted to the NICUs in Zagazig University Hospital, ${ }^{11}$ Benha University Hospital ${ }^{25}$ and Tanta City Hospitals ${ }^{26}$ were $19.2,30.6$, and $27.7 \%$ (of the studied neonates), respectively. In our study, higher morbidity and mortality rates due to congenital anomalies may be explained by the increased referral of such cases from other health care centers, since there is a neonatal surgical unit considered as a referral unit for critically ill neonates with surgical diseases. Also, management of congenitally abnormal neonates needs advanced facilities and skills, which are not available sometimes in many centers. Similar to our work, Fahmy et al $^{12}$ in Cairo University hospital, Egypt found that the majority of their inborn deaths were preterm (78.48\%), also deaths were significantly more among inborn than outborn (37.2 vs. $17.2 \%, p<0.001$ ). This could be due to the increasing number of inborn hospital admissions for preterm babies and also that admitted babies' status in outborn NICU is better, with favorable outcome. Omoigberale et $\mathrm{al}^{27}$ reported that the high cost of caring for a newborn may be responsible for delay in presentation as well as delay in administering care, resulting in poor neonatal outcome.

In our study, the commonest reason for mortality among premature newborns was RDS with a case fatality rate of $56.7 \%$, that was matched with results of studies conducted by Verma et $a^{16}$ and Raikwar. ${ }^{28}$ These studies stated that the commonest causes of death were prematurity with RDS, sepsis, and perinatal asphyxia. On the contrary, Abd el aziz et $\mathrm{al}^{11}$ found that sepsis (63.41\%) followed by respiratory failure (26.82\%), and cardiogenic shock (14.63\%) were the commonest cause of death among studied neonates, Their results also agreed with studies done by Muthukumaran, ${ }^{29}$ and Medhat and Khashana. ${ }^{30}$

In the current study, multiple congenital anomalies were the main reason of death among inborn and outborn admitted full-term infants, with case fatality rates of 67.4 and $42.6 \%$, respectively, followed by perinatal asphyxia, congenital intestinal obstruction, meconium aspiration syndrome, and sepsis. Andegiorgish et $\mathrm{al}^{31}$ found that congenitally 
malformed neonates were three times more likely to die than their counterparts, similar to other research in which most neonates with congenital malformation died. ${ }^{31}$

In the present study, the morbidity and mortality caused by neonatal sepsis was low. Only 23 cases with sepsis were recorded. This low number could be due to solid infection control measures applied at our hospital, with increased awareness among health care providers. Moreover, sepsis could be underestimated in this study because septic cases were limited to only those diagnosed at admission. The difference between our study and others, in Egypt and India, regarding high neonatal morbidity and mortality rates is explained by the fact that our hospital as previously described, is considered a high referral center for surrounding governorates. Besides patients in complicated forms due to delayed presentations and poor management, the poverty in Upper Egypt, high cost of caring for a newborn, and low educational level have also contributed to bad outcomes in our locality. In our study, there were no confirmed neonates with COVID-19; instead, three asymptomatic cases were suspected of having COVID-19 due to positive maternal infection but were found to be negative after laboratory examination. According to Wang et al, ${ }^{32}$ there is no definite evidence of intrauterine vertical transmission of SARSCoV-2, but more high-quality research is needed. Although virological and serological evidence is useful in clarifying this issue, scientifically sound and trustworthy assays should be utilized, and newborns should be followed for 6 to 18 months after birth to draw meaningful conclusions when serological results are employed.

For assessing the effectiveness of treatment given in a hospital environment, knowing the admission outcomes is critical. Among NICUs in various parts of the world, NMRs vary dramatically. This disparity is most likely due to differences in the attending population, antenatal treatment, admission requirements, unique exclusion and inclusion criteria, and neonatal care given.

\section{Limitation of Study}

This study was performed at a tertiary care and referral hospital where most of the patients were critically ill and complicated. Thus, results of our work may not reflect the true morbidity and mortality burden prevalent in the community as a whole.

\section{Conclusion}

RDS, prematurity, and congenital malformations were significantly linked with neonatal mortality in Neonatal Care Unit of our hospital. For controlling local neonatal deaths, one should prioritize early management of low birth weight, preterm births, and neonatal complications. The most common causes of neonatal deaths are preventable and treatable; careful assessment and risk detection are the best ways to minimize neonatal mortality in our community. The incidence of congenital malformations in our resource-limited setting is high and antenatal diagnosis rates are very low.

\section{Recommendations}

This study revealed that the majority of inborn admissions were preterm babies and RDS was the commonest cause of morbidity and mortality in these babies. Thus, this study recommends administration of antenatal corticosteroid therapy for women at risk of preterm birth from 24 to 34 weeks of gestation, starting continuous positive airway pressure therapy at diagnosis, and early surfactant replacement therapy (within first 2 hours after birth) for preterm neonates with established RDS. To improve the mortality for neonates with congenital anomalies, this study recommends referral of mothers who are suspected to deliver a baby with extreme low birth weight $(<1,000 \mathrm{~g})$, anomalies needing immediate surgical interventions, and life-threatening anomalies to a hospital with level IV NICU (University Hospitals). Regarding outborn neonatal admissions, access to high-quality and timely care for preterm and sick newborns are critical to improving outcomes. The goal is to reduce neonatal mortality and morbidity when the management of a sick infant exceeds care level ability provided in a district hospital. Communication with the referral center must be done prior to transport to ensure availability of beds and services required for babies (e.g., surfactant, surgery). Provision of ambulance services with trained staff for newborn referrals could improve health outcomes of preterm and sick newborns. Sensitization and training of health care providers on national referral protocols/guidelines, setting expectations for adherence, government investments in newborn referral systems, and standardizing initiating and receiving facility referral communication, are all urgently needed. Upgrading provision of newborn care at lower-level facilities will decrease referral load at higher-level facilities.

\section{Authors' Contribution}

S.M.A.-A. carried the study design, examined cases, and shared in the writing of manuscript. E.A.H. was involved in the selection of cases, data collection and entry, validation, and coding, as well as shared in writing the manuscript. A.M. S. was involved in selection of cases, data collection, and shared in writing the manuscript and gathering references. All authors have read and approved the manuscript for publication.

\section{Funding \\ None}

Conflict of Interest

None declared.

\section{References}

1 Parasher V, Khatri R, Yadav S, Mittal U. The pattern of admission and their related outcomes in NICU of a tertiary care teaching hospital, Udaipur, Rajasthan, India. Pediatr Rev-Int J Pediatr Res 2021;8(01):16-22

2 Hug L, Alexander M, You D, Alkema LUN Inter-agency Group for Child Mortality Estimation. National, regional, and global levels and trends in neonatal mortality between 1990 and 2017, with 
scenario-based projections to 2030: a systematic analysis. Lancet Glob Health 2019;7(06):e710-e720

3 King BC, Mowitz ME, Zupancic JAF. The financial burden on families of infants requiring neonatal intensive care. Semin Perinatol 2021;45(03):151394

4 Belachew A, Tewabe T, Dessie G. Neonatal mortality and its association with antenatal care visits among live births in Ethiopia: a systematic review and meta-analysis. J Matern Fetal Neonatal Med 2020;26:1-8. Doi: 10.1080/14767058. 2020.1718093

5 Wardlaw T, You D, Hug L, Amouzou A, Newby H. UNICEF Report: enormous progress in child survival but greater focus on newborns urgently needed. Reprod Health 2014;11(01):82

6 Organization WH. World Health Statistics 2019: Monitoring Health for the SDGs, Sustainable Development Goals. 2019

7 Organization WH. Every Newborn: An Action Plan to End Preventable Deaths. 2014

8 Bishop D, Dyer RA, Maswime S, et al; ASOS investigators. Maternal and neonatal outcomes after caesarean delivery in the African Surgical Outcomes Study: a 7-day prospective observational cohort study. Lancet Glob Health 2019;7(04):e513-e522

9 Muhe LM, McClure EM, Mekasha A, et al. A prospective study of causes of illness and death in preterm infants in Ethiopia: the SIP study protocol. Reprod Health 2018;15(01):116

10 Falciglia HS, Merkel RC, Glover V, Hasselfeld KA, Brady WK. The mortality of periviable and extremely premature infants and their impact on the overall neonatal mortality rate. Sci Rep 2020;10 (01):2503

11 Abd El Aziz T, Elbanna E, Sarhan D, Ibrahim SE. Clinical characterizations of neonatal admission in neonatal intensive care unit at Zagazig University Children Hospital. Zagazig Univ Med J 2020;26 (05):775-786

12 Fahmy N, Ramy N, El Houchi S, et al. Outborns or inborns: clinical audit of the two intensive care units of Cairo University Hospital. Egyptian Pediatric Association Gazette. 2017;65(01):10-14

13 Tekleab AM, Amaru GM, Tefera YA. Reasons for admission and neonatal outcome in the neonatal care unit of a tertiary care hospital in Addis Ababa: a prospective study. Res Rep Neonatol 2016;6:17-23

14 El-Zanati F. Egypt Health Issues Survey, Ministry of Health and Population, El-Zanaty and Associates and, ICF International. International Central Agency for Public Mobilisation and Statistics Report issued by: Economic and Social Justice Unit: Knowledge and Prevalence of Hepatitis B and C. 2015:25-50

15 Bokade CM, Meshram RM. Morbidity and mortality patterns among outborn referral neonates in central India: Prospective observational study. J Clin Neonatol 2018;7(03):130-135

16 Verma J, Anand S, Kapoor N, Gedam S, Patel U. Neonatal outcome in newborns admitted in NICU of tertiary care hospital in central India: a 5-year study. Int J Contemp Pediatrics 2018;5(04):1364-1367

17 Ochoga M, Abah R, Michael A, et al. Retrospective assessment of neonatal morbidity and mortality in the special care baby unit of a private health facility in Benue State, North Central Nigeria. Niger J Paediatr 2020;47(04):353-357

18 Ekwochi U, Ndu IK, Nwokoye IC, Ezenwosu OU, Amadi OF, Osuorah D. Pattern of morbidity and mortality of newborns admitted into the sick and special care baby unit of Enugu State University Teaching Hospital, Enugu state. Niger J Clin Pract 2014;17(03): 346-351

19 Seboka J, Abera AM, Rebitu AD. Pattern of admission and outcome of neonate admitted to Adama Hospital Medical College Neonatal Intensive Care Unit, Adama, Ethiopia. Int J Sci Basic Appl Res 2018; 42(01):59-71

20 Ike Elizabeth U, Modupe O0. Pattern of diseases and care outcomes of neonates admitted in special care baby unit of University College Hospital, Ibadan, Nigeria from 2007 to 2011. J Nurs Health Sci 2015;4(01):62-71

21 Baseer KAA, Mohamed M, Abd-Elmawgood EA. Risk factors of respiratory diseases among neonates in neonatal intensive care unit of Qena University Hospital, Egypt. Ann Glob Health 2020;86 (01):22

22 Elizabeth U, Oyetunde M. Pattern of diseases and care outcomes of neonates admitted in IOSR. J Nurs Heal Sci Ver I. 2015; 4:1940-2320

23 Eze P, Al-Maktari F, Alshehari AH, Lawani LO. Morbidities \& outcomes of a neonatal intensive care unit in a complex humanitarian conflict setting, Hajjah Yemen: 2017-2018. Confl Health 2020;14(01):53

24 Aijaz N, Huda N, Kausar S. Disease burden of NICU, at a tertiary care hospital, Karachi. J Dow Univ Health Sci 2012;6(01):32-35

25 Hady S, Haie O, Eldesouky R, Gabal M. Incidence and the underlying factors of neonatal mortality in the university hospital of Benha, Egypt. Int J Med Health Sci 2016;5(04):237-244

26 El-Ganainy HFR, El-Mashad AE-RM, Shihab NS, Abu-Hamama AM. Risk factors for neonatal mortality in neonatal intensive care units in Tanta City. Egypt J Hosp Med 2019;75(01):1996-2006

27 Omoigberale AI, Sadoh WE, Nwaneri DU. A 4 year review of neonatal outcome at the University of Benin Teaching Hospital, Benin City. Niger J Clin Pract 2010;13(03):321-325

28 Raikwar P. A study of neonatal admission pattern and outcome from rural Haryana. Glob J Res Anal 2018;19:12-16

29 Muthukumaran N. Mortality profile of neonatal deaths and deaths due to neonatal sepsis in a tertiary care center in southern India: a retrospective study. Int J Contemp Pediatrics 2018;5(04): 1583-1587

30 Medhat $\mathrm{H}$, Khashana A. Incidence of neonatal infection in South Sinai, Egypt. Int J Infect 2017;4(01):e36615

31 Andegiorgish AK, Andemariam M, Temesghen S, Ogbai L, Ogbe Z, Zeng L. Neonatal mortality and associated factors in the specialized neonatal care unit Asmara, Eritrea. BMC Public Health 2020; 20(01):10

32 Wang C, Zhou YH, Yang HX, Poon LC. Intrauterine vertical transmission of SARS-CoV-2: what we know so far. Ultrasound Obstet Gynecol 2020;55(06):724-725 